\title{
Queixas de tontura em indivíduos com hipotireoidismo congênito em idade escolar e suas implicações nas habilidades acadêmicas e comunicativas
}

\author{
Dizziness complaints in school-aged individuals with congenital hypothyroidism and \\ the implications for academic and communication skills
}

\author{
Elen Pereira de Jesus ${ }^{1}$, Caio Leônidas Oliveira de Andrade², Crésio de Aragão Dantas Alves ${ }^{3 *}$ \\ ${ }^{1}$ Fonoaudióloga pela União Metropolitana de Educação e Cultura (UNIME) e Mestranda do Programa de Pós- \\ graduação Processos Interativos dos Órgãos e Sistemas da Universidade Federal da Bahia (UFBA); ${ }^{2}$ Professor \\ do Curso de Graduação em Fonoaudiologia da Universidade do Estado da Bahia (UNEB), Mestre e Doutor pelo \\ Programa de Pós-graduação Processos Interativos dos Órgãos e Sistemas da Universidade Federal da Bahia (UFBA); \\ ${ }^{3}$ Doutor em Medicina e Saúde pela UFBA, Professor Associado de Pediatria da Faculdade de Medicina da Bahia da \\ UFBA e do Programa de Pós-graduação Processos Interativos dos Órgãos e Sistemas da UFBA
}

\begin{abstract}
Resumo
Introdução: o hipotireoidismo congênito $(\mathrm{HC})$ pode causar danos nas estruturas do sistema vestibular ocasionando sintomas de tontura que pode acarretar em implicações nas habilidades linguísticas e acadêmicas dos indivíduos. Objetivo: verificar a ocorrência de queixas de tontura em crianças com $\mathrm{HC}$ e as suas implicações nos aspectos comunicativos e desempenho acadêmico por meio de uma entrevista estruturada com os cuidadores. Metodologia: estudo exploratório de caráter descritivo, seccional, realizado no município de Salvador, sendo incluídos crianças de ambos os sexos, com diagnóstico de hipotireoidismo congênito e idade entre 5 a 10 anos. Os dados foram coletados entre 2014 a 2018 em um Serviço de Referência em Triagem Neonatal no Estado da Bahia. Para avaliar as variáveis qualitativas foi utilizado o teste qui-quadrado e para as variáveis quantitativas foi utilizado o teste t-student, adotando nível de significância de 5\%. Resultados: os indivíduos com disgenesia apresentaram uma frequência de $64 \%$ de queixas relacionadas a tontura. Verificou-se uma frequência considerável de dificuldade nas competências de escrita e com perfil comunicativo de maior relevância para a habilidade de conversação por meio de frases curtas. Conclusão: os achados sugerem que a presença de queixas de tontura nos os indivíduos com $\mathrm{HC}$ pode predispor a insucessos nos aspectos acadêmicos e comunicativos.

Palavras-chave: Hipotireoidismo Congênito. Tontura. Desempenho Acadêmico. Comunicação.
\end{abstract}

\begin{abstract}
Introduction: congenital hypothyroidism $(\mathrm{CH})$ can damage the structures of the vestibular system, causing dizziness symptoms that can have implications for the linguistic and academic skills of individuals. Objective: to verify the occurrence of dizziness complaints in children with $\mathrm{CH}$ and its implications for communicative aspects and academic performance through a structured interview with caregivers. Methodology: an exploratory, descriptive, sectional study carried out in the city of Salvador, including children of both sexes, diagnosed with congenital hypothyroidism and aged between 5 and 10 years. Data were collected between 2014 and 2018 at a Neonatal Screening Reference Service in the State of Bahia. To assess qualitative variables, the chi-square test was used and for quantitative variables, the t-student test was used, adopting a significance level of 5\%. Results: individuals with dysgenesis had a frequency of $64 \%$ of complaints related to dizziness. There was a considerable frequency of difficulty in writing skills and with a communicative profile of greater relevance to the ability to converse through short sentences. Conclusion: the findings suggest that the presence of complaints of dizziness in individuals with $\mathrm{CH}$ may predispose to failures in academic and communicative aspects. Keywords: Congenital hypothyroidism. Dizziness. Academic achievement. Communication.
\end{abstract}

\section{INTRODUÇÃO}

Dentre as endocrinopatias mais comuns no recém-nascido, o hipotireoidismo congênito (HC) configura-se como o de maior incidência, podendo ocasionar danos irreversíveis para o funcionamento adequado do siste-

Correspondente/Corresponding: *Crésio de Aragão Dantas Alves - Instituto de Ciências da Saúde, Universidade Federal da Bahia - Av. Reitor Miguel Calmon S/N - Vale do Canela Salvador-Ba, CEP: 40110-100 - Tel: (71) 99178-4055 - E-mail: cresio.alves@uol.com.br ma nervoso central (SNC) (ROVET, 2002) e estruturas associadas, como o sistema vestibular, se não tratado precocemente.

Em modelo animal, há evidências clínicas de que a ausência e/ou privação dos hormônios tireoidianos (HT) em idades precoces do desenvolvimento podem gerar alteração na morfogênese dos receptores vestibulares (MEYERHOFF, 1979; DECHESNE; LEGRAND; SANS, 1984) e na atividade dos canais semiciculares (DECHESNE; LEGRAND; SANS, 1984), o que demonstra intrínseca 
influência desses hormônios na homeostase da função vestibular. Diante do desenvolvimento anormal das estruturas cocleovestibulares, podemos verificar alterações importantes envolvendo o equilíbrio estático e dinâmico.

Essas funções vestibulares integradas a outros sistemas são responsáveis pela manutenção adequada da postura (GANANÇA; CAOVILLA, 1999; ALMEIDA, 2009). Para que o equilíbrio aconteça, é necessária a interação das vias vestibulares, tronco cerebral e cerebelo, que permitam ao sistema nervoso central (SNC) reconhecer posições e movimentos da cabeça em relação ao corpo e ao espaço (DOUGLAS, 2002).

Disfunções dos mecanismos relacionados ao sistema de equilíbrio corporal podem interferir na vida diária dos indivíduos, especialmente quando os seus sinais e sintomas ocorrem em idades precoces, podendo acarretar prejuízos nos aspectos linguísticos, psicológica e no desempenho escolar (GUARDIOLA; FERREIRA; ROTTAN, 1998).

Devido à dificuldade que crianças na idade escolar apresentam para expressar de forma correta os sintomas, é possível que elas confundam tonturas com mal-estar sem causa aparente (BITTAR et al., 2002), dificultando a percepção desses problemas pelas crianças, pais e cuidadores, o que torna esses casos de difícil registro subnotificados (BATHIA et al., 1977; BITTAR, 2003; COAVILLA et al., 2000; ERBEK et al., 2006).

Diante do exposto, este estudo tem como objetivo verificar a ocorrência de queixas de tontura no público pediátrico com $\mathrm{HC}$ e as suas implicações nos aspectos comunicativos e desempenho acadêmico por meio de uma entrevista estruturada com os cuidadores.

\section{METODOLOGIA}

Trata-se de um estudo exploratório de caráter descritivo, seccional, com amostra obtida por conveniência, realizado entre 2014 a 2018. A casuística foi composta por 39 crianças, de ambos os sexos, com diagnóstico de $\mathrm{HC}$ e idade entre 5 a 10 anos, que compareceram regularmente, em datas pré-agendadas, para o atendimento médico em um Serviço de Referência em Triagem Neonatal no Estado da Bahia.

Nessa análise, os pais ou responsáveis foram consultados como fonte das informações a fim de ratificar a presença dos sintomas relatados pelas crianças. Adotaram-se os seguintes critérios de exclusão para a pesquisa: ser portador de síndromes, doenças neurológicas ou psiquiátricas; apresentar alteração na inspeção do meato acústico externo; ter histórico de doenças de orelha média e/ou externa, fatores de risco para deficiência; relatar doenças infecciosas atuais ou pregressas envolvendo o sistema nervoso central; apresentar outras doenças metabólicas, bem como qualquer outra forma de hipotireoidismo que não seja de caráter congênito e permanente. Por causa do foco de intervenção em interações mãe-criança ou cuidador/responsável-criança no tratamento de reposição hormonal, outras pessoas que não faziam parte do convívio da criança não foram incluídas na pesquisa.

Como instrumentos de pesquisa, foram utilizados três protocolos. O primeiro teve como critério incluir o indivíduo na amostra deste estudo, consistindo, basicamente, em uma investigação prévia sobre a presença de fatores de risco para deficiência auditiva, histórico de outras doenças e antecedentes familiares. Para coletar os dados referentes às queixas de tontura foi utilizado um questionário estruturado composto por perguntas fechadas com questões específicas sobre a presença ou ausência da tontura/vertigem nas crianças com HC. Para a aplicação dos protocolos, os pais ou cuidadores foram submetidos ao processo formal de entrevista.

Os dados clínicos e laboratoriais dos pacientes foram extraídos do prontuário médico, com o registro da dosagem do hormônio tireoestimulante (TSH) e tiroxina livre $\left(T_{4} L\right)$, pelo método de eletroquimioluminescência, bem como a idade cronológica, idade do rastreio neonatal e de início do tratamento do $\mathrm{HC}$, sendo os valores de referências destas duas últimas conforme preconizados pelo Ministério da Saúde, na Portaria (GM/MS) no 822/0113.

A análise estatística usou o software computacional SPSS (versão 21.0). As variáveis contínuas foram descritas por valores médios, desvios-padrão e frequências absolutas e relativas. A análise bivariada entre variáveis categóricas foi realizada a partir do teste qui-quadrado. Para amostras independentes, foi utilizado o teste t-student para comparação de médias entre dois grupos, adotando nível de significância de $5 \%(p \leq 0,05)$.

Foram respeitadas as normas do Comitê de Ética em Pesquisa com Seres Humanos da Universidade do Estado da Bahia, sob o parecer no 3.454.114/2019, de acordo com o Conselho Nacional de Saúde Resolução 466/2012. Todos os sujeitos da pesquisa assinaram o Termo de Assentimento Livre e Esclarecido (quando cabível), e o Termo de Consentimento Livre e Esclarecido (TCLE) foi assinado por seus responsáveis.

\section{RESULTADOS}

Participaram do estudo 39 indivíduos portadores de $\mathrm{HC}$ em idade escolar ( $x=7.9, \mathrm{DP} \pm 3,1)$, sendo que mais da metade (64\%) era do sexo feminino. A queixa de tontura foi mais frequente nos indivíduos que demonstraram níveis séricos de TSH mais elevados durante os testes do pezinho e exames confirmatórios, assim como no aumento da idade diagnóstica, no entanto sem significância estatística. (Tabela 1). 
Tabela 1- Distribuição das medidas de tendência central e valores máximos, mínimos e percentis relacionados aos aspectos clínicos-laboratoriais dos indivíduos com HC com e sem tontura ( $n=39)$.

\begin{tabular}{|c|c|c|c|c|c|c|c|c|c|c|c|c|c|}
\hline \multirow{3}{*}{$\begin{array}{c}\text { Aspectos } \\
\text { Clínicos }\end{array}$} & \multicolumn{6}{|c|}{ Ausência de queixa de tontura } & \multicolumn{6}{|c|}{ Presença de queixa de tontura } & \multirow{3}{*}{$\begin{array}{c}\text { Valor } \\
\text { de } \\
\text { P.* }\end{array}$} \\
\hline & \multirow{2}{*}{$\begin{array}{l}\text { Média } \\
\text { (DP } \pm)\end{array}$} & \multirow{2}{*}{ Min. } & \multicolumn{3}{|c|}{ Percentis } & \multirow{2}{*}{ Máx. } & \multirow{2}{*}{$\begin{array}{l}\text { Média } \\
\text { (DP } \pm)\end{array}$} & \multirow{2}{*}{ Min. } & \multicolumn{3}{|c|}{ Percentis } & \multirow{2}{*}{ Máx. } & \\
\hline & & & 25 & 50 & 75 & & & & 25 & 50 & 75 & & \\
\hline Id. TP (dias) & $28,5(26,3)$ & 5 & 9,3 & 21 & 40,3 & 104 & $21,9(15,0)$ & 4 & 9 & 23 & 27 & 53 & 0,452 \\
\hline TSH TP & $78,2(86,2)$ & 0,4 & 19 & 44,8 & 98,2 & 324 & $181(211,5)$ & 30,8 & 38,7 & 71,3 & 324,1 & 701 & 0,068 \\
\hline Id. diag. (dias) & $63,1(31,2)$ & 30 & 40 & 57,5 & 66,5 & 133 & $98,3(156,6)$ & 19 & 26,8 & 50,5 & 107,5 & 627 & 0,333 \\
\hline TSH diag. & $72,2(73,1)$ & 0,9 & 15,1 & 40,8 & 120 & 300,6 & $127(125)$ & 9,6 & 35,1 & 120 & 120 & 439 & 0,115 \\
\hline Id. atual & $7,92(3,12)$ & 5 & 6 & 7 & 9 & 17 & $8,13(2,8)$ & 5 & 7 & 7 & 9 & 16 & 0,828 \\
\hline
\end{tabular}

Legenda: DP士: Desvio Padrão / Min.: Minimo / Máx.: Máximo / Id. TP (dias): Idade no teste do pezinho em dias / TSH TP: Nível sérico do hormônio tireoestimulante no teste do pezinho / Id. diag. (dias): Idade diagnóstica em dias / TSH diag.: Nível sérico do hormônio tireoestimuante no diagnóstico. Id. Atual: Idade atual, criança já em processo de segmento hormonal.

*Teste T Student

Fonte: Dados da pesquisa

O gráfico 1 mostra que os indivíduos com disgenesia apresentaram uma frequência de $64 \%$ de queixas relacionadas a tontura quando comparados às crianças com não-disgenesia (28\%) e os eutiroidianas (0\%).

Gráfico 1 - Distribuição da frequência da presença de queixas de tontura nos indivíduos com hipotireoidismo congênito nos diferentes grupos etiológicos da doença $(n=39)$.

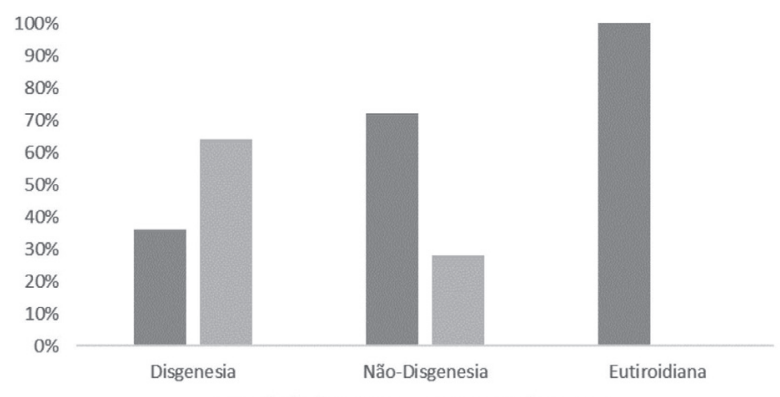

- Ausência de tontura Presença de tontura

Fonte: Dados da pesquisa

A queixa de tontura foi mais prevalente no sexo feminino (80\%) conforme ilustra o gráfico 2 .

Gráfico 2 - Distribuição da ocorrência de queixa de tontura nos diferentes gêneros $(n=39)$.

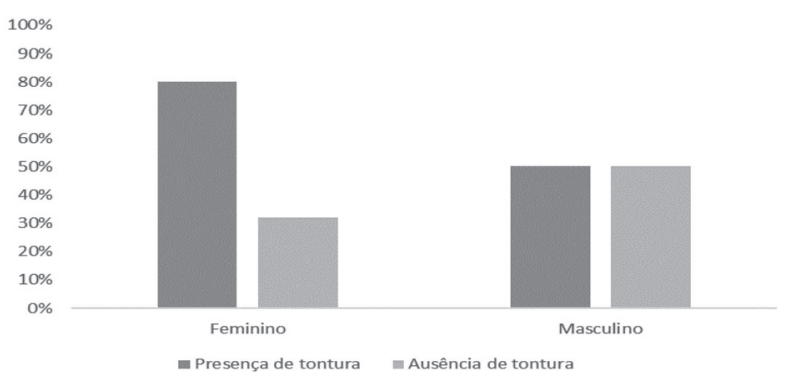

Fonte: Dados da pesquisa
O gráfico 3 mostra a percepção de zumbido nas crianças com $\mathrm{HC}$ com e sem tontura.

Gráfico 3 -Distribuição da frequência da percepção do zumbido nos indivíduos com hipotireoidismo congênito com e sem tontura $(n=39)$.

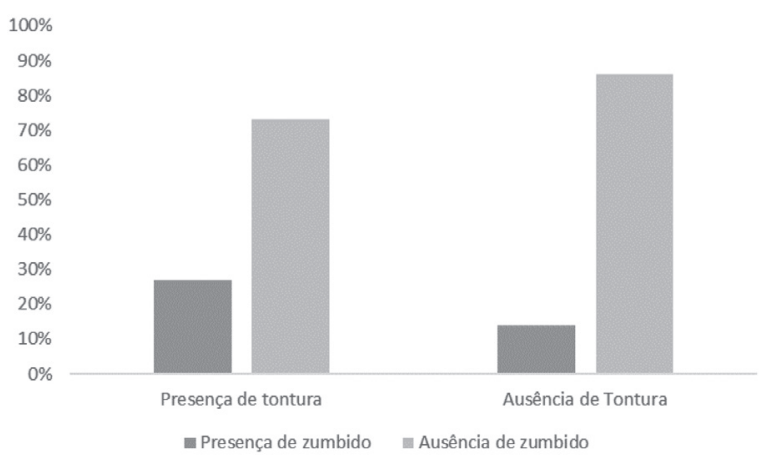

Fonte: Dados da pesquisa

No que tange as habilidades de leitura, fala e escrita, verificou-se uma frequência considerável de dificuldade nas competências de escrita em relação às demais habilidades nos indivíduos com queixa de tontura. (Tabela 2).

Tabela 2 -.Distribuição da frequência da queixa tontura e sua relação com dificuldade de escrita, leitura e fala $(n=39)$.

\begin{tabular}{ll|l|l|l|l|l}
\hline \multirow{2}{*}{$\begin{array}{c}\text { Queixa de } \\
\text { tontura }\end{array}$} & \multicolumn{2}{c}{$\begin{array}{c}\text { Dificuldade } \\
\text { de escrita }\end{array}$} & \multicolumn{2}{c}{$\begin{array}{c}\text { Dificuldade } \\
\text { de leitura }\end{array}$} & \multicolumn{2}{c}{$\begin{array}{c}\text { Dificuldade } \\
\text { de fala }\end{array}$} \\
\cline { 2 - 8 } & $\begin{array}{c}\text { sim } \\
\text { (\%) }\end{array}$ & $\begin{array}{c}\text { não } \\
\text { (\%) }\end{array}$ & $\begin{array}{c}\text { sim } \\
\text { (\%) }\end{array}$ & $\begin{array}{c}\text { não } \\
\text { (\%) }\end{array}$ & $\begin{array}{c}\text { sim } \\
\text { (\%) }\end{array}$ & $\begin{array}{c}\text { não } \\
\text { (\%) }\end{array}$ \\
\hline Ausência & 33,3 & 66,7 & 21,7 & 78,3 & 45,5 & 54,5 \\
\hline Presença & 66,7 & 33,3 & 23,1 & 76,9 & 48,3 & 41,7 \\
\hline Valor de P.* & \multicolumn{2}{c}{0,053} & \multicolumn{2}{c}{0,180} & \multicolumn{2}{c}{0,623} \\
\hline
\end{tabular}

Legenda: Dif. De escrita: Dificuldade de escrita / Dif. De leitura: Dificuldade de leitura / Dif. De fala: Dificuldade de fala.

*Teste qui-quadrado 
O gráfico 4 ilustra o comportamento do perfil comunicativo das crianças com $\mathrm{HC}$ com e sem queixa de tontura. $\mathrm{O}$ grupo com queixa de tontura apresentou perfil comunicativo com maior relevância para a habilidade de conversação por meio de frases curtas.

Gráfico 4- Queixa de tontura correlacionado ao perfil comunicativo geral.

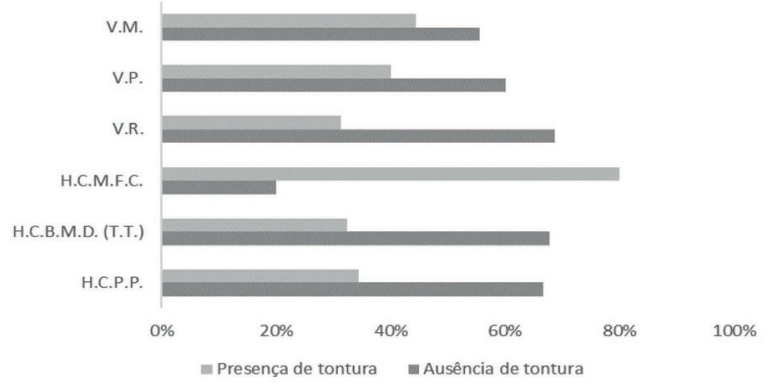

Legenda: V.M.: Vocabulário mediano / V.P.: Vocabulário pobre / V.R. Vocabulário rico / H.C.M.F.C.: Habilidade de conversação por meio de frases curtas / H.C.B.M.D. (T.T.): Habilidade de conversação com boa manutenção do diálogo (troca de turno) / H.C.P.P.: Habilidade de conversação com poucas palavras.

Fonte: Dados da pesquisa

\section{DISCUSSÃO}

Foi possível observar que os indivíduos com HC que apresentaram níveis séricos do TSH piores durante o período do teste do pezinho e, posteriormente, no exame confirmatório apresentaram maior ocorrência da presença de queixas de tontura. Esses achados também foram evidenciados quando analisada a idade em que foi realizada a confirmação da doença, onde os indivíduos com a idade diagnóstica tardia também demonstraram maiores ocorrências de queixas de tontura.

A literatura especializada adverte quanto aos prejuízos dos indivíduos com $\mathrm{HC}$ que apresentam alterações nos níveis séricos dos $\mathrm{HT}$, assim como, para aqueles indivíduos que iniciaram o tratamento de reposição hormonal tardio (ANDROVANDI; NUNES, 2004; BARGAGNA et al., 2000; BARGAGNA et al., 2006; BRAGATTI-WINCKLER; ROTTA, 1994; GERJÃO; LAMONICA, 2008; KEMPERS et al., 2006; OERBECK et al., 2003; OERBECK et al., 2005; ROVET, 2005). Para o Ministério da Saúde (2004), a idade preconizada para o teste do pezinho deve ocorrer entre o quinto ao sétimo dia de vida e estabelece que o início do tratamento para o $\mathrm{HC}$ deve ser até o $28^{\circ}$ dia.

Esses cuidados são fundamentais para que sejam prevenidas as sequelas irreversíveis no organismo dos indivíduos. Situação que pode explicar os achados de maiores queixas de tontura relacionadas no $\mathrm{HC}$ que apresentaram baixa adesão no segmento hormonal, corroborando com a hipótese de que a atividade da função vestibular é diretamente dependente dos níveis adequados dos $\mathrm{HT}$, fato que comprova que esse público constitui um potencial de risco para disfunções vestibulares (ANDRADE, 2014).

Os efeitos nocivos das alterações dos HT no ouvido interno não são bem fundamentadas, os estudos ainda são escassos e com metodologias mais voltadas para modelo animal, os quais evidenciam que a redução ou ausência dos níveis séricos desses hormônios exerce efeitos deletérios nas estruturas e funcionalidades das vias periféricas e centras do sistema vestibular. (ANDRADE, 2017; BATHIA et al., 1977; BITTAR et al., 2003; FRIAUF et al., 2008).

Nos casos graves da doença, onde o $\mathrm{HC}$ se manifesta nas formas etiológicas da disgenesia, o indivíduo pode cursar com injúrias no sistema nervoso central SNC (RAMOS et al., 2003) e, mais especificamente, nas funções do ouvido interno (LICHTENBERGER-GESLIN et al., 2013). Isso pode explicar a notória ocorrência de queixas vestibulares em crianças diagnosticadas com algumas das formas de disgenesia, como apontada no resultado desse estudo.

Adicionalmente, a diferença de gênero parece influenciar a maior ocorrência de sintomas de tontura. Os achados mostraram uma frequência de $80 \%$ das crianças do sexo feminino com queixas de tontura. É provável que o fato do estudo ser composto por uma amostra predominantemente do sexo feminino tenha influenciado nesses resultados. No entanto, a literatura especializada aponta que mulheres, por apresentarem variações hormonais ao longo da vida, estão sujeitas à maior incidência desses sintomas (BITTAR, 1997).

Da mesma forma, o grupo dos indivíduos com HC que cursaram com queixas de tontura tinham maior ocorrência de zumbido (27\%) quando comparados ao grupo de indivíduos com $\mathrm{HC}$ sem queixas de tontura (14\%), o que pode ser sugestivo de indícios de comprometimento dos sistemas coclear e/ou vestibular (SANTOS et al., 2010).

As disfunções do sistema vestibular em crianças, tais como queixas e sintomas de tontura, podem afetar o desempenho escolar, sendo, portanto, o histórico de constantes fracassos escolares um sinal de alerta para prováveis prejuízos dessas vias sensoriais (GANANÇA; COAVILLA, 1977; SARTORI; PANHOCA, 2007). O presente estudo também evidenciou que, nos indivíduos com HC que apresentaram queixas de tontura, uma considerável frequência de relatos de dificuldades nas habilidades relacionadas à escrita e fala, em respectivo.

Esses prejuízos podem estar diretamente relacionados a uma falha na função do sistema proprioceptivo com o vestibular, os quais juntos controlam a postura corporal, a oculomotricidade, coordenação motora, o equilíbrio corporal e a percepção viso-espacial, mecanismos essenciais para a aprendizagem da linguagem falada e escrita (CAMPOS et al., 1996).

Há relatos na literatura sobre destacam a presença a existência de defasagem na habilidade auditiva expres- 
siva, auditiva receptiva e visual, assim como, alterações dos níveis de linguagem (fonológica, fonética, pragmática, semântica, léxical e morfossintática) (BARGAGNA et al., 2000; BARGAGNA et al., 2006; LAMÔNICA; GEJÃO; SILVA, 2006).

Embora os achados do presente artigo tenham demonstrado a influência dos sintomas da tontura nos aspectos comunicativos e acadêmicos nos indivíduos com $\mathrm{HC}$, a sua metodologia precisa ser replicada em outros estudos com uma amostra randomizada e delineamento de estudo prospectivo a fim de confirmar tais achados que foram coletados exclusivamente por meio de relatos de cuidadores daquelas crianças portadoras do HC, fato que constitui a principal limitação desse estudo.

Também é importante que novos estudos sejam realizados por meio de avaliação objetiva desses indivíduos no intuito de comprovar tais queixas relacionadas a tontura, bem como traçar o real topodiagnóstico das prováveis afecções no sistema vestibular e detectada a disfunção vestibular.

\section{CONCLUSÃO}

Os achados sugerem que a presença de queixas de tontura nos os indivíduos com $\mathrm{HC}$ podem os predispor a insucessos nos aspectos acadêmicos e comunicativos.

\section{REFERÊNCIAS}

ALMEIDA, R. P. et al. Avaliação Auditiva de Crianças com Hipotireoidismo Congênito. RPBS, Fortaleza, v. 22, n. 1, p. 41-47, 2009.

ANDRADE, C.L.O. Estudo das emissões otoacústicas produto por distorção em indivíduos com hipotireoidismo congênito em tratamento com levotiroxina. 2014. 97 f. Dissertação (Mestrado em Processos Interativos dos Órgãos e Sistemas) - Instituto de Ciências da Saúde, Universidade Federal da Bahia, Salvador, 2014.

ANDRADE, C.L.O. et al. Prevalência de sintomas otoneurológicos em indivíduos com hipotireoidismo congênito: estudo piloto. Cad. saúde colet., Rio de Janeiro, v. 25, n. 2, p. 144-151, abr. 2017.

ANDROVANDI, C.; NUNES M.L.T. Avaliação intelectual de escolares com hipotireoidismo congêntio. Aletheia, Canoas, v. 20, p. 55-64, jul./dez. 2004.

BARGAGNA, S. et al. Neuropsychological follow-up in early-treated congenital hypothyroism: a problem-oriented approach. Thyroid, New York, v. 10, n. 3, p. 243-249, Mar. 2000.

BARGAGNA, S. et al. Neurophychiatric outcome in patients with congenital hypothyroidism precautiously treated: risk factors analysis in a grup of patients from Tuscany. Minerva Pediatr., Torino, v. 58, n. 3, p. 279-287, June. 2006.

BATHIA, P.L. et al. Audiological and vestibular function tests in hypothyroidism. Laryngoscope, St. Louis, v. 87, n. 12, p.2082-2099, Dec. 1977.

BITTAR, R.S.M. et al. Prevalência das alterações metabólicas em pacientes portadores de queixasvestibulares. Rev. Bras. Otorrinolaringol., Rio de Janeiro, v. 69, n. 1, p.64-68, jan./fev. 2003.

BITTAR, R.S.M. et al. Reabilitação vestibular na criança: estudo preliminar. Rev. Bras. Otorrinolaringol., Rio de Janeiro, v. 68, n. 4, p. 496-499, jul./ago. 2002.
BRAGATTI-WINCKLE, M. I.; ROTTA, N. T. Hormônios tireóideos na sintomatologia de doenças neuropsiquiátricas. Arq. Neuro-Psiquiatr. Porto Alegre, v. 52, n. 3, p. 427-430, 1994.

BRASIL. Ministério da Saúde. Secretaria de Atenção à Saúde. Departamento de Atenção Especializada. Manual de normas técnicas e rotinas operacionais do programa nacional de triagem neonatal. 2. ed. Brasília: MS, 2004.

CAMPOS, M.l. et al. Prevalência de sinais de disfunção vestibular em crianças com vertigem e/ou outros tipos de tontura. RBM-ORL, [s.I], v. 3, p. 165-170, 1996.

CAOVILLA, H. H. et al. Vertigem paroxística benígna da infância. In: SILVA, M.L. et al. Quadros clínicos otoneurológicos mais comuns. São Paulo: Atheneu, 2000. p. 109-117.

DECHESNE, C.; LEGRAND, C.; SANS, A. Effects of experimental hypothyroid is mon the surfacestructures of vestibular receptors in developing rats. Rev. Laryngol. Otol. Rhinol., Bordeaux, v.15, p. 237-241, 1984.

DOUGLAS, C. R. Fisiologia do equilíbrio. In: DOUGLAS, C.R. Tratado de fisiologia aplicada à fonoaudiologia. 5. ed. São Paulo: Rode, 2002.

ERBEK, S. H. et al. Vertigo in childhood: a clinical experience. IJPORL, [s.I], v. 70, n. 9, p. 1547-1554, Oct. 2006.

FRANCO, E. S.; PANHOCA, I. Avaliação otoneurológica em crianças com queixa de dificuldades escolares: pesquisa da função vestibular. Rev. Bras. Otorrinolaringol., São Paulo, v. 73, n. 6, p. 803-815, dez. 2007.

FRIAUF, E. et al. Hypothyroidism impairs chloride homeostasis and onset of inhibitory neurotransmission in developing auditory brainstem and hippocampal neurons. Eur. J. Neurosci.. Oxford, v. 28, n. 12, p. 2371-2380, 2008.

GANANÇA, M. M.; CAOVILLA, H. H. Labirintopatia na infância. In: CALDAS, N.; NETO, S. C.; SIH, T. Otologia e audiologia em pediatria. Rio de Janeiro: Revinter Ltda, 1999. p. 277-286.

GEJÃO, M. G.; LAMONICA, D. A. C. Habilidades do desenvolvimento em crianças com hipotireoidismo congênito: enfoque na comunicação. PróFono R. Atual. Cient. Barueri, v. 20, n. 1, p. 25-30, mar. 2008.

GUARDIOLA, A.; FERREIRA, L. T.; ROTTA, N. T. Associação entre desempenho das funções corticais e alfabetização em uma amostra de escolares de primeira série de Porto Alegre. Arq Neuro-Psiquiatr, Porto Alegre, v. 56, n. 2, p. 281-288, 1998.

KEMPERS, M. J. E. et al. Intellectual and motor development of young adults with congenital hypothyroidism diagnosed by neonatal screening. JCEM, [s.I], v. 91, n. 2, p. 418-424, 2006.

LAMÔNICA, D. A. C; GEJÃO, M. G.; SILVA, G. K. Linguagem receptiva e vocabulário de crianças com hipotireoidismo congênito. Rev. Soc. Bras. Fonoaudiol., São Paulo, Suplemento Especial, p. 57, 2006.

LICHTENBERGER-GESLIN, L. et al. Factors associated with hearing impairment in patients with congenital hypothyroidism treated since the neonatal period: a national population based study. JCEM, [s.I], v. 98, n. 9, p. 3644-3652, 2013.

MEYERHOFF, W. L. Hypothyroidism and the ear: electrophysiological, morphological and chemical considerations. Laryngoscope, St. Louis, v. 89, p. $1-25,1979$.

OERBECK, B. et al. Congenital hypothyroism: influence of disease severity and I-thyroxine treatment on intellectual, motor, and schoolassociated outcomes in young adults. Pediatrics, Evanston, v. 112, n. 4, p. 923-930, Oct. 2003.

OERBECK B. et al. Congenital hypothyroism: no adverse effects of high 
dose throxine treatment on adult memory, attention, and behaviour. Arch. Dis. Child., London, v. 90, n. 2, p. 132-137, Feb. 2005.

RAMOS, A. J. S. et al. Avaliação do programa de rastreamento de doenças congênitas em Campina Grande PB, Brasil. ABEM. São Paulo, v. 47 , n. 3, p. 280-284, jun. 2003.

ROVET, J. F. Children with congenital hypothyroidism and their siblings: do they really differ? Pediatrics, Evanston, v. 115, n. 1, p. 52-57, Jan. 2005.

ROVET, J. F. Congenital hypothyroidism: an analysis of persisting deficits and associated factors. Child Neuropsychol., Lisse, v. 8, n. 3, p. 150162, Sept. 2002.

Submetido em: 02/11/2020

Aceito em: 05/11/2020 